\title{
Closely linked non-additive blood pressure quantitative trait loci
}

\author{
Edward J. Toland - Yasser Saad · Shane Yerga-Woolwine • Steven Ummel • \\ Phyllis Farms · Ramona Ramdath · Bryan C. Frank - Norman H. Lee · \\ Bina Joe
}

Received: 26 November 2007 / Accepted: 4 January 2008

(C) Springer Science+Business Media, LLC 2008

\begin{abstract}
There is enough evidence through linkage and substitution mapping to indicate that rat chromosome 1 harbors multiple blood pressure (BP) quantitative trait loci (QTLs). Of these, BP QTL1b was previously reported from our laboratory using congenic strains derived by introgressing normotensive alleles from the LEW rat onto the genetic background of the hypertensive Dahl salt-sensitive (S) rat. The region spanned by QTL1b is quite large (20.92 Mb), thus requiring further mapping with improved resolution so as to facilitate systematic identification of the underlying genetic determinant(s). Using congenic strains containing the LEW rat chromosomal segments on the Dahl salt-sensitive (S) rat background, further iterations of congenic substrains were constructed and characterized. Collective data obtained from this new iteration of congenic substrains provided evidence for further fragmentation of QTL1b with improved resolution. At least two separate genetic determinants of blood pressure underlie QTL1b. These are within $7.40 \mathrm{Mb}$ and $7.31 \mathrm{Mb}$ and are known as the QTL1b1 region and the QTL1b2 region, respectively. A
\end{abstract}

E. J. Toland and Y. Saad contributed equally to this work.

Electronic supplementary material The online version of this article (doi:10.1007/s00335-008-9093-1) contains supplementary material, which is available to authorized users.

E. J. Toland · Y. Saad · S. Yerga-Woolwine - S. Ummel ·

P. Farms $\cdot$ R. Ramdath $\cdot$ B. Joe $(\bowtie)$

Physiological Genomics Laboratory, Department of Physiology and Pharmacology, University of Toledo College of Medicine, 3035 Arlington Avenue, Toledo, OH 43614, USA

e-mail: bina.joe@utoledo.edu

B. C. Frank · N. H. Lee

Department of Pharmacology and Physiology, George

Washington University, Washington, DC 20037, USA genetic interaction was detected between the two BP QTLs. Interestingly, five of the previously reported differentially expressed genes located within the newly mapped QTL1b1 region remained differentially expressed. The congenic strain S.LEW(D1Mco36-D1Mco101), which harbors the QTL1b1 region alone but not the QTL1b2 region, serves as a genetic tool for further dissection of the QTL1b1 region and validation of $N r 2 f 2$ as a positional candidate gene. Overall, this study represents an intermediary yet obligatory progression towards the identification of genetic elements controlling BP.

\section{Introduction}

Genetic analysis of quantitative traits in rodent models is viewed as a suitable alternate approach to facilitate the identification of genetic determinants of complex polygenic traits in humans (Cowley 2006; Deng 2007; Flint and Mott 2001). Blood pressure (BP) is one such complex polygenic trait. Multiple mapping studies in rat models have provided compelling evidence for the existence of BP quantitative trait loci (QTLs) (Garrett et al. 2005; Joe and Garrett 2005; Joe et al. 2003; Lee et al. 2006; Saad et al. 2007a, b), some of which are located in regions homologous to human chromosomes that are linked to BP (Cowley 2006; Stoll et al. 2000). While such colocalization of QTLs controlling BP in both rats and humans is intriguing, mapping with improved resolution is required to ultimately reveal the identities of the underlying genetic determinants. The present study is focused on achieving this objective on a previously identified blood pressure QTL (QTL1b) on rat chromosome 1 (Saad et al. 2001), also documented as "BP94" on the Rat Genome Database (http://www.rgd.mcw.edu). 
Congenic strains derived by introgressing normotensive alleles from the Lewis rat onto the hypertensive genetic background of the Dahl salt-sensitive (S) rat were used to develop a new iteration of congenic substrains. Data from this new panel of congenic substrains demonstrated that there are at least two genetic determinants of BP within the previously mapped QTL1b. Further dissection of one of these fragmented QTLs, QTL1b1 is prioritized. This QTL region contained five differentially expressed genes as previously reported. This previous study was conducted with the $\mathrm{S}$ rat and a congenic strain with a larger introgressed segment, i.e., S.LEW(D1Mco36-D1Rat49) (Joe et al. 2005). The status of gene expression of these positional candidates of QTL1b1 as assessed by comparing the gene expression of $\mathrm{S}$ with the congenic strain that defines the limits of QTL1b1, i.e., S.LEW(D1Mco36-D1Mco101), is also presented.

\section{Materials and methods}

\section{Animals}

The inbred Dahl salt-sensitive (SS/Jr or S) rats were from our colony. The Lewis (LEW/NCrlBR) rats, originally obtained from Charles River Laboratories (Wilmington, MA), were maintained in our animal facility and are referred to as LEW. All congenic substrains were constructed as per previously published procedures (Meng et al. 2003). S.LEW (D1Mco36-D1Rat49) is the progenitor strain for the congenic substrains S.LEW(D1Mco36-D1Rat131a), S.LEW (D1Mco36-D1Rat131b), S.LEW(D1Mco36-D1Mco77), S.LEW (D1Mco36-D1Rat106), S.LEW(D1Mco36-D1Mco101), and S.LEW(D1Mco99-D1Rat49).

\section{Microsatellite markers}

New microsatellite markers were developed from sequences identified by (1) comparative mapping of the rat sequence with the Celera mouse genomic sequence (http://www.celera.com) or (2) searching the rat genome sequence data available (RGSC 3.4, December 2004) through the Ensembl database (http://www.ensembl.org). Sequence information of the newly identified polymorphic markers with the prefix D1Mco is available at our website, http://www.hsc.utoledo.edu/depts/physiology/research/rat/ marker.html.

\section{Genotyping}

Congenic substrain DNA was extracted from a tail biopsy using the QIAamp Tissue Kit (Qiagen, Chartsworth, CA). PCR-based genotyping with microsatellite markers was done using standard techniques described previously (Gu et al. 1996).

\section{Phenotyping}

Rats were weaned at 30 days of age and maintained on a low-salt $(0.3 \% \mathrm{NaCl})$ Harlan Teklad diet 7034 (Madison, WI). In experiments comparing a congenic strain with $\mathrm{S}$ rats, 20 or 30 male congenic substrain rats were used. They were matched by age and weight with an equal number of male $\mathrm{S}$ control rats, and the animals were caged such that each cage contained a congenic strain and an $S$ rat. In experiments comparing two congenic strains with $\mathrm{S}$ rats, the housing of each congenic strain was either with one $S$ rat or with one rat representing the other congenic strain. At 40-42 days of age, the rats were fed a $2 \% \mathrm{NaCl}$ Harlan Teklad diet, TD94217, for 24 days. Systolic blood pressure was measured using the tail-cuff method on conscious restrained rats warmed to $28^{\circ} \mathrm{C}$ using semiautomatic equipment from IITC (Woodland Hills, CA). BP was measured once a day for four consecutive days. The BP value of each day was the average of two to three consistent readings. The final $\mathrm{BP}$ value used was the average $\mathrm{BP}$ value of the 4 days. Rats were euthanized with $\mathrm{CO}_{2}$. Final body and heart weights were measured and recorded. Furthermore, in an effort to increase the sample size for testing using the tail-cuff method, the following design was adapted. This method spans 4 days of testing 90 rats for BP by two operators. Day 1: 30 animals of the congenic strain S.LEW(D1Mco36-D1Mco101) were tested with $15 \mathrm{~S}$ rats; Day 2: 30 animals of the congenic strain S.LEW(D1Mco36-D1Rat106) were tested with $15 \mathrm{~S}$ rats; Day 3 is a repeat of Day1; and Day 4 is a repeat of Day 2. At the end of the study, $10 \mathrm{~S}$ rats and 10 S.LEW(D1Mco36-D1Mco101) rats were surgically implanted with C-40 transmitters (Data Sciences International, St. Paul, MN) as described previously (Joe et al. 2003). After 5 days of recovery, the transmitters were turned on and real-time systolic BP, diastolic BP, mean BP, heart rate, and pulse pressure were monitored and recorded over 4 days. Average BP values of $4 \mathrm{~h}$ were used for statistical analysis.

\section{Quantitative real-time PCR}

Kidney RNA samples from 40-42-day-old S $(n=8)$ and S.LEW(D1Mco36-D1Mco101) $(n=8)$ animals that were fed a low -salt $(0.3 \%)$ diet were collected and analyzed by real-time PCR on an ABI PRISM ${ }^{\circledR} 7700$ Sequence Detection System (Applied Biosystems, Foster City, CA), as described previously (Joe et al. 2005). Briefly, total RNA was reversed transcribed using random hexamer primers. The resulting cDNA was diluted and used as 
template for quantitative PCR using SYBR Green. PCR primers were selected for specificity by NCBI BLAST of the rat genome, and amplicon specificity was verified by first-derivative melting curve analysis using software provided by PerkinElmer/Applied Biosystems. Quantitation and normalization of relative gene expression was accomplished using the comparative $\mathrm{C}_{\mathrm{T}}$ method or $\Delta \Delta \mathrm{C}_{\mathrm{T}}$. $\Delta \Delta \mathrm{C}_{\mathrm{T}}$ values were converted into ratios by $2^{-\Delta \Delta \mathrm{CT}}$ and averaged across biological replicates. The expression of the "housekeeping" genes malate dehydrogenase (RefSeq accession number NM_012600), glutamate dehydrogenase (NM_012570), and hydroxysteroid sulfotransferase (XM_001081762) was used for normalization as these genes did not exhibit differential expression in our microarray assays. Sequence of primers used for the real-time RT-PCR are given in Supplementary Table 1.

\section{Statistical analysis}

For each congenic substrain tested, the following variables were collected: body weight $(\mathrm{g})$, systolic BP $(\mathrm{mmHg})$, heart weight $(\mathrm{mg})$, and relative heart weight (the ratio of heart weight to final body weight). An independent-samples $t$ test ( $\mathrm{S}$ vs. a single congenic substrain) or a one-way analysis of variance (ANOVA) with the Tukey post-hoc test of comparisons ( $\mathrm{S}$ vs. 2 congenic substrains) was done using SPSS software (SPSS, Inc., Chicago, IL). In experiments where a significant difference in body weight between $\mathrm{S}$ and a congenic strain was observed, heart weight was corrected for differences in body weight by regression of heart weight on body weight. The corrected heart weight values were used in place of the measured values for those comparisons. Genetic interactions of the BP effects were tested by a two-way ANOVA $(2 \times 2$ ANOVA) using SPSS software. BP effect of each experimental rat in a study was computed by subtracting the mean $\mathrm{BP}$ of all the $\mathrm{S}$ rats in the study from the BP of the experimental rat. This normalized BP effect was then comparable between experiments and was used for the $2 \times 2$ ANOVA.

\section{Results}

The genetic tools for this study were the panel of S.LEW congenic substrains that are schematically represented in Fig. 1. These were constructed by screening $F_{2}$ populations derived by crossing $\mathrm{S}$ with the parental congenic strain. BP QTL1b was previously located within the open bar shown toward the left-hand side of the diagram between the markers D1Rat35 and D1Rat131. To recapitulate, the QTL1b region was inferred as the differential segment between the congenic strain with the BP effect,
S.LEW(D1Rat35-D1Rat49), and the congenic strain without the BP effect, S.LEW(D1Mco87-D1Rat71) (Saad et al. 2001) (Fig. 1). In the present study, this localization was further confirmed by two of the new congenic substrains, S.LEW(D1Mco36-D1Rat131a) and S.LEW(D1Mco36D1Rat131b), both spanning QTL1b and significantly lowering $\mathrm{BP}$ of the $\mathrm{S}$ by 17 and $24 \mathrm{mmHg}$, respectively (Fig. 1, Table 1). Heart weights and relative heart weights of these two congenic strains were also significantly lower than the S, corroborating the BP-lowering effect (Table 1). Congenic substrains S.LEW(D1Mco36-D1Mco77), S.LEW(D1Mco36-D1Rat106), and S.LEW(D1Mco36D1Mco101), with shorter introgressed LEW segments from the top end of the QTL, also significantly lowered BP of the $\mathrm{S}$ rat by 19,25 , and $25 \mathrm{mmHg}$ (Fig. 1, Table 1). Multiple experiments with these strains indicated that the heart weight data corroborated the observed changes in BP between each congenic strain and $\mathrm{S}$ (Table 1). Together, these data suggest that a BP QTL could be traced within the limits of the congenic substrain with the shortest introgressed segment, S.LEW(D1Mco36-D1Mco101). The region spanned by this QTL, called the QTL1b1 region, is $7,397,670 \mathrm{bp}$ from the marker D1Mco36 to marker D1Mco101. This is a conservative localization because the upper limit of the QTL1b1 region could very well be defined by the proximal end of S.LEW(D1Rat35-D1Rat49) as opposed to the top end of S.LEW(D1Mco36D1Mco101). However, to arrive at this conclusion, further evidence will be required to demonstrate that the differential top ends of S.LEW(D1Rat35-D1Rat49) and S.LEW(D1Mco36-D1Mco101) (genomic region between D1Mco36 and D1Mco49) do not contain BP QTLs.

Among the congenic substrains shown in Fig. 1, note that congenic substrain S.LEW(D1Mco85-D1Rat49), which does not contain the QTL1b1 region, also significantly lowers BP of the $\mathrm{S}$ rat by $13 \mathrm{mmHg}$. This suggests that S.LEW(D1Mco85-D1Rat49) contains a genetic determinant of BP, which is clearly not the QTL1b1 region. This second QTL identified within the original QTL1b was labeled the QTL1b2 region (Fig. 1). Note that a segment of the introgressed LEW alleles within S.LEW(D1Mco85D1Rat49) was also present within the congenic region of the substrain S.LEW(D1Mco99-D1Rat49), which did not have a significantly lower BP or relative heart weight compared to $\mathrm{S}$ (Fig. 1, Table 1). This localized the QTL1b2 region to the proximal portion of the congenic region that was not shared between S.LEW(D1Mco85D1Rat49) and S.LEW(D1Mco99-D1Rat49) (Fig. 1). This interval spans 7,309,286 bp between the microsatellite markers D1Mco85 and D1Rat106. It is important to note that between the localizations of QTL1b1 and QTL1b2, the localization of QTL1b1 is better because the introgressed region of the congenic strain that "traps" QTL1b1 is much 


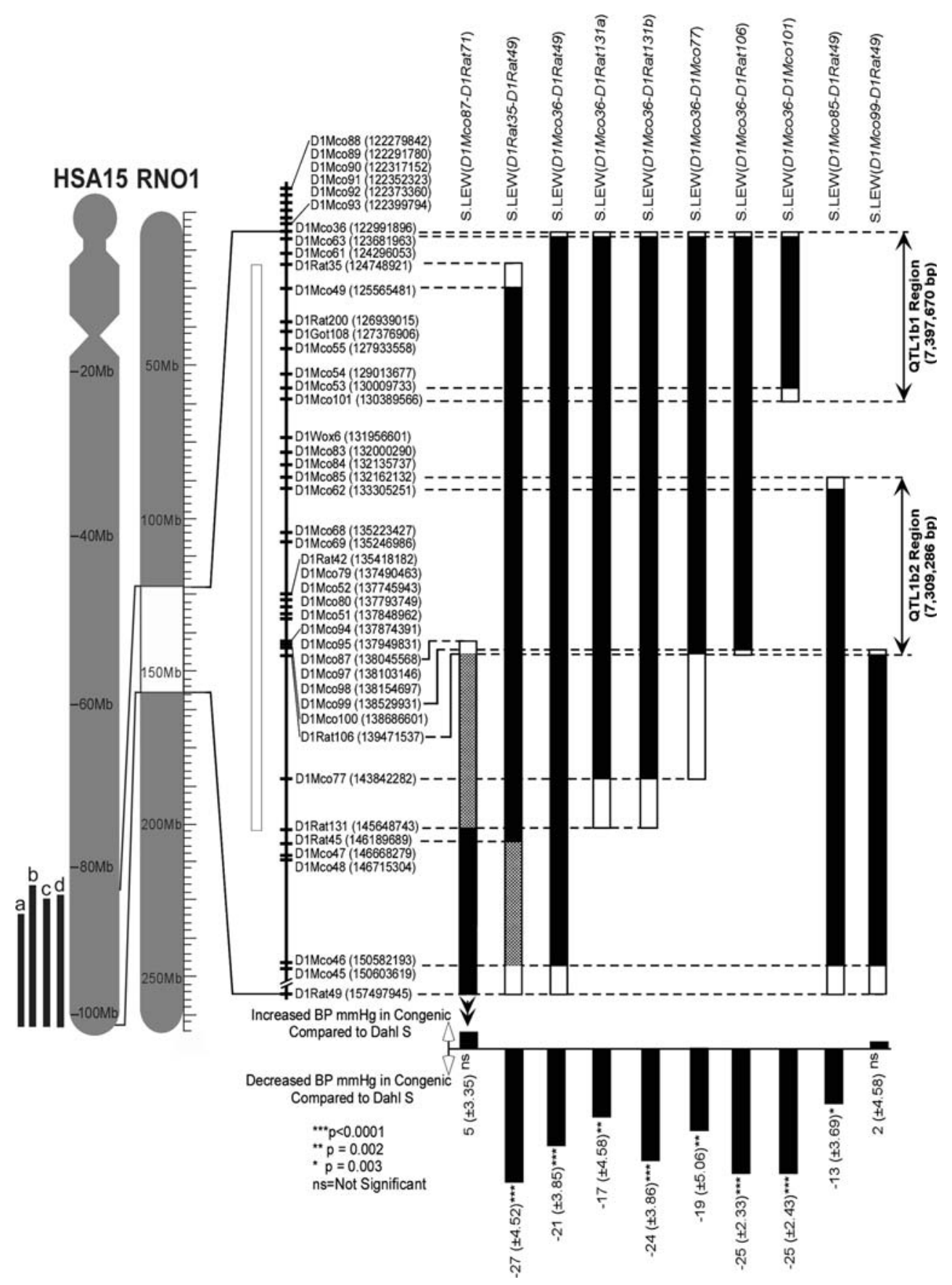

Fig. 1 Schematic representation of substitution mapping of BP QTL1b using congenic substrains. Black solid bars to the left of the diagram show the location of orthologous regions of human chromosome 15 containing BP QTLs: References are (a) Krushkal et al. 1999; (b) Weder et al. 2003; (c) Xu et al. 1999a; (d) Xu et al. 1999b. Homology of this region to rat chromosome 1 is represented by the clear segment within the shaded representation of rat chromosome 1 (RNO1). The open bar to the left of the physical map denotes the previously mapped BP QTL1b (Saad et al. 2001). The physical map of the segment of RNO1 in focus and the location of the microsatellite markers are drawn to scale. Details of the newly identified polymorphic markers with the prefix D1Mco are available at our website (http://www.hsc.utoledo.edu/depts/physiology /research/rat/marker.html). Congenic strains are shown as black bars flanked by white bars. Flanking white bars are regions containing recombinant breakpoints. The crosslined congenic segment toward the distal portion of S.LEW(D1Rat35-D1Rat49) and proximal portion of S.LEW(D1Mco87-D1Rat71) represents heterozygous SL genotype. Underneath each congenic substrain, the BP effect in comparison with $\mathrm{S}$ is shown. Values for BP are the mean effect with the SE in parenthesis. Data from S.LEW(D1Mco87-D1Rat71), S.LEW(D1Rat35-D1Rat49), S.LEW(D1Mco36-D1Rat49), and S.LEW(D1Mco85-D1Rat49) are previously published strains (Saad et al. 2001), the regions of recombination of which are further genotyped using new markers and presented here for completeness. Double arrow pointing downward of S.LEW(D1Mco87-D1Rat71) indicates that the introgressed region shown is incomplete. To the extreme right of the diagram are two double-headed arrows that depict the newly mapped QTL1b1 region and QTL1b2 region 
Table 1 Effects of rat chromosome 1 congenic strains on blood pressure, body weight, heart weight, and relative heart weight

\begin{tabular}{|c|c|c|c|c|c|c|c|c|}
\hline Congenic strain & \multicolumn{4}{|c|}{ Blood pressure $(\mathrm{mmHg})$} & \multicolumn{3}{|c|}{ Body weight (g) } & $p$ value \\
\hline S.LEW(D1Mco87-D1Rat71) ${ }^{\mathrm{a}}$ & $202[1.72]$ & $207[2.87]$ & $+5(3.35)$ & 0.106 & $318[3.57]$ & $329[2.27]$ & $+11(4.23)$ & 0.023 \\
\hline S.LEW(D1Rat35- D1Rat49) ${ }^{\mathrm{a}}$ & $222[3.70]$ & $195[2.87]$ & $-27(4.52)$ & $<0.0001$ & $309[3.43]$ & 304 [2.97] & $-5(5.03)$ & 0.603 \\
\hline S.LEW(D1Mco36- D1Rat49) ${ }^{\mathrm{a}}$ & 209 [2.97] & $188[3.08]$ & $-21(3.85)$ & $<0.0001$ & 314 [1.72] & $307[3.23]$ & $-7(3.78)$ & 0.187 \\
\hline S.LEW(D1Mco36-D1Rat131a) & $195[3.27]$ & $178[2.22]$ & $-17(4.58)$ & 0.002 & $314[2.23]$ & $305[2.40]$ & $-9(3.08)$ & 0.007 \\
\hline S.LEW(D1Mco36-D1Rat131b) ${ }^{b}$ & $194[3.38]$ & $170[2.33]$ & $-24(3.86)$ & $<0.0001$ & $309[2.53]$ & $306[1.91]$ & $-3(3.08)$ & 0.576 \\
\hline S.LEW(D1Mco36-D1Mco77) & $203[2.98]$ & $184[3.07]$ & $-19(5.06)$ & 0.002 & $345[1.65]$ & $334[2.40]$ & $-11(3.50)$ & 0.009 \\
\hline S.LEW(D1Mco36-D1Rat106) ${ }^{\mathrm{c}}$ & $198[1.78]$ & $173[1.74]$ & $-25(2.33)$ & $<0.0001$ & $328[2.61]$ & $335[2.61]$ & $+7(3.50)$ & 0.118 \\
\hline S.LEW(D1Mco36-D1Mco101) ${ }^{\mathrm{b}}$ & $198[1.78]$ & $173[1.36]$ & $-25(2.43)$ & $<0.0001$ & $328[2.61]$ & $323[2.25]$ & $-5(4.42)$ & 0.561 \\
\hline S.LEW(D1Mco85- D1Rat49) & 209 [2.97] & $196[1.93]$ & $-13(3.69)$ & 0.003 & $314[1.72]$ & $316[2.87]$ & $+2(3.62)$ & 0.817 \\
\hline \multirow[t]{2}{*}{ S.LEW(D1Mco99-D1Rat49) } & $195[3.27]$ & $197[3.99]$ & $+2(4.58)$ & 0.874 & $314[2.23]$ & $302[1.89]$ & $-12(3.08)$ & 0.001 \\
\hline & \multicolumn{4}{|c|}{ Heart weight $(\mathrm{g})$} & \multicolumn{4}{|c|}{ Relative heart weight } \\
\hline S.LEW(D1Mco87-D1Rat71) ${ }^{\mathrm{a}}$ & $1.21[0.023]$ & $1.29[0.018]$ & $+0.08(0.029)$ & 0.006 & $3.83[0.037]$ & $3.88[0.034]$ & $+0.05(0.051)$ & 0.361 \\
\hline S.LEW(D1Rat35- D1Rat49) ${ }^{\mathrm{a}}$ & $1.31[0.016]$ & $1.25[0.127]$ & $-0.06(0.020)$ & 0.007 & $4.32[0.057]$ & $4.13[0.044]$ & $-0.19(0.069)$ & 0.021 \\
\hline S.LEW(D1Mco36- D1Rat49) ${ }^{a}$ & $1.25[0.015]$ & $1.15[0.017]$ & $-0.10(0.022)$ & $<0.0001$ & $3.99[0.042]$ & $3.74[0.038]$ & $-0.25(0.054)$ & $<0.0001$ \\
\hline S.LEW(D1Mco36-D1Rat131a) & $1.25[0.008]$ & $1.18[0.010]$ & $-0.07(0.014)$ & $<0.0001$ & $4.04[0.029]$ & $3.84[0.033]$ & $-0.20(0.047)$ & $<0.0001$ \\
\hline S.LEW(D1Mco36-D1Rat131b) ${ }^{\mathrm{b}}$ & $1.20[0.006]$ & $1.11[0.014]$ & $-0.09(0.013)$ & $<0.0001$ & $3.89[0.023]$ & $3.60[0.045]$ & $-0.29(0.045)$ & $<0.0001$ \\
\hline S.LEW(D1Mco36-D1Mco77) & $1.35[0.009]$ & $1.29[0.010]$ & $-0.06(0.016)$ & 0.002 & $3.95[0.029]$ & $3.80[0.033]$ & $-0.15(0.047)$ & 0.01 \\
\hline S.LEW(D1Mco36-D1Rat106) ${ }^{\mathrm{c}}$ & $1.30[0.013]$ & $1.24[0.013]$ & $-0.06(0.017)$ & 0.002 & $3.98[0.030]$ & $3.71[0.031]$ & $-0.27(0.041)$ & $<0.0001$ \\
\hline S.LEW(D1Mco36-D1Mco101) ${ }^{\mathrm{b}}$ & $1.30[0.013]$ & $1.24[0.011]$ & $-0.06(0.022)$ & 0.006 & $3.98[0.030]$ & $3.82[0.023]$ & $-0.16(0.052)$ & 0.008 \\
\hline S.LEW(D1Mco85- D1Rat49) & $1.25[0.152]$ & $1.21[0.136]$ & $-0.04(0.020)$ & 0.007 & $3.99[0.042]$ & $3.83[0.031]$ & $-0.16(0.051)$ & 0.011 \\
\hline S.LEW(D1Mco99-D1Rat49) & $1.25[0.008]$ & $1.27[0.010]$ & $+0.02(0.014)$ & 0.413 & $4.04[0.029]$ & $4.14[0.037]$ & $+0.10(0.047)$ & 0.096 \\
\hline
\end{tabular}

Values in square brackets are standard error of the mean. Values in parentheses are standard error of the mean difference

${ }^{a}$ Data from these strains were previously published (Saad et al. 2001) and are presented here for completeness

${ }^{b}$ Combined data of two independent experiments

${ }^{c}$ Combined data of three independent experiments. The BP of the S phenotyped along with S.LEW(D1Mco36-D1Rat106) and the BP of the S phenotyped along with S.LEW(D1Mco36-D1Mco101) were not statistically different from each other. Therefore, data analysis was combined for S vs. S.LEW(D1Mco36-D1Rat106) and S.LEW(D1Mco36-D1Mco101)

${ }^{\mathrm{d}}$ Effect $=$ Congenic value $-\mathrm{S}$ value. A negative number in the Effect column indicates a decrease in a trait in the congenic strain compared to the $\mathrm{S}$ rat, whereas a positive number indicates an increase in a trait of the congenic strain compared to the $\mathrm{S}$ rat. Number of rats in each group was 20, except for the study comparing S.LEW(D1Mco36-D1Mco101), S.LEW(D1Mco36-D1Rat106), and S rats, in which 30 rats from each group were tested concomitantly. All rats tested were males

shorter than that of the congenic strain that "traps" QTL1b2. The region labeled QTL1b2 indicates that it is a prioritized region for further dissection based on cumulative evidence from multiple strains.

Data presented in Fig. 1 show that the LL alleles of QTL1b1 reduced BP by $25 \mathrm{mmHg}$ [represented by the congenic strain S.LEW(D1Mco36-D1Mco101)] and that the LL alleles at QTL1b2 reduced BP by $13 \mathrm{mmHg}$ [represented by S.LEW(D1Mco85-D1Rat49)]. If the two QTLs were additive, then, at least theoretically, in a strain that contains LL alleles at QTL1b1 and QTL1b2 one would expect a BP lowering effect of $25+13=38 \mathrm{mmHg}$. However, this is not the case. Note that none of the strains in Fig. 1 show a BP effect of $38 \mathrm{mmHg}$. Therefore, an interactive effect was suspected. A $2 \times 2$ ANOVA of the $\mathrm{BP}$ effects of the QTL1b1 region and the QTL1b2 region revealed a statistically significant variance in BP that was indeed due to an interaction between the two BP QTLs $(p<0.01)$ (Fig. 2). This interaction resulted in interference between the BP effects of the two QTLs. In other words, the observed net BP effect of LL alleles, when present at both the BP QTLs, was statistically smaller than the expected net BP effect of the two QTLs had they been statistically additive.

Because the QTL1b1 region harbors Nr2f2, which is a prioritized positional candidate for BP QTL1b (Joe et al. 2005), we have chosen to focus on the QTL1b1 region for further genetic dissection. $N r 2 f 2$ was prioritized as one of the differentially expressed positional candidate genes because a network of perturbations in gene expression was trackable that was related to the potential functionality of $N r 2 f 2$ in BP regulation (Joe et al. 2005). The observed 


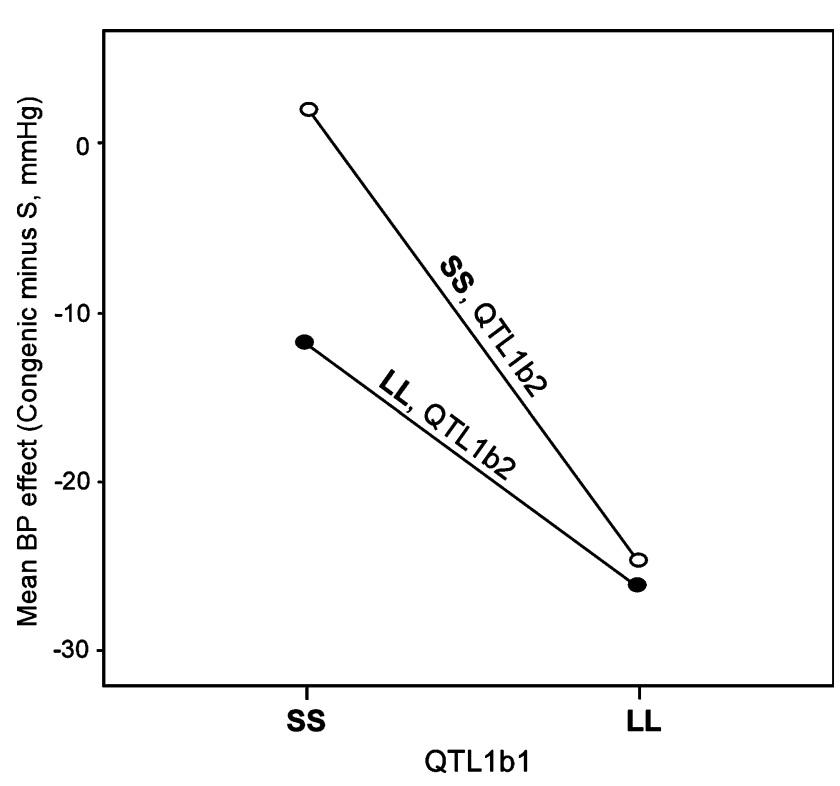

Fig. 2 Genetic interaction between BP QTL1b1 region and BP QTL1b2 region analyzed by two-way analysis of variance. The graph illustrates the $2 \times 2$ ANOVA results obtained by comparing BP effects of S.LEW(D1Mco36-D1Rat106), S.LEW(D1Mco36D1Mco101), S.LEW(D1Mco85-D1Rat49), and S.LEW(D1Mco99D1Rat49). BP effects of all individual animals input into the $2 \times 2$ ANOVA was obtained as described in the Materials and methods section. Genotypes at the QTL1b1 region are on the $x$ axis. The SS genotype of the QTL1b2 region is represented by the open circles and the LL genotype of the QTL1b2 region is represented by the closed circles. Nonparallel lines are indicative of a genetic interaction $(p<0.01)$

differential expression of $N r 2 f 2$ was identical between lowand high-salt-fed animals. The BP QTL effect of the congenic strain S.LEW(D1Mco36-D1Mco101) encompassing $N r 2 f 2$ was also compared with the $\mathrm{S}$ by using telemetry (Fig. 3). The results from telemetry were consistent with those obtained by the tail-cuff method. Systolic BP of S rats $(211 \pm 2.05 \mathrm{mmHg})$ was $33 \mathrm{mmHg}$ higher than that of S.LEW(D1Mco36-D1Mco101) (178 $\pm 1.56 \mathrm{mmHg}$, $p<0.0001$ ) (Fig. 3). Similarly, diastolic BP and pulse pressure of $\mathrm{S}$ rats were also significantly higher than that of the congenic strain $(15 \mathrm{mmHg}, p<0.001$, and $10 \mathrm{mmHg}$, $p<0.001$, respectively). The heart rates of $\mathrm{S}$ rats, however, were not significantly different from that of the S.LEW(D1Mco36-D1Mco101) (Fig. 3).

Previously, using a custom QTL-interval-specific oligonucleotide array and a whole-genome rat cDNA array, we identified five differentially expressed positional candidates within the newly mapped QTL1b1 region (Joe et al. 2005). Interestingly, all of these genes remain differentially expressed between $\mathrm{S}$ and the congenic strain that harbors a shorter LEW segment with a BP-lowering effect (Table 2). The direction of change in gene expression remained unaltered for three candidate genes, chromodomain helicase DNA binding protein $2(C h d 2)$ and the novel genes
X62953 and XM_980342, but it was opposite for two other candidate genes, i.e., a hypothetical protein (BE108294) and a transcription factor ( $N r 2 f 2)$ (Table 2). The expression of the latter two genes was lower in the congenic strain S.LEW(D1Mco36-D1Mco101) compared with that in the $\mathrm{S}$, whereas it was higher in the congenic strain S.LEW(D1Mco36-D1Rat49) compared with that in the S (Joe et al. 2005). This is intriguing and suggests that the direction of change in expression of positional candidate genes such as $N r 2 f 2$ should be closely monitored for association with BP in future iterations of mapping. It is also interesting to note that seven out of the nine other transcription factors that are located elsewhere on the genome and known to cross-talk with $N r 2 f 2$ remain differentially expressed in the same direction between $\mathrm{S}$ and S.LEW(D1Mco36-D1Mco101) (Table 2) as was previously described (Joe et al. 2005). Overall, the data suggest that based on the property of differential gene expression of positional candidates, multiple genes remain as candidates for the BP QTL1b1.

\section{Discussion}

Considerable attention has been paid to rat chromosome 1 because of the BP QTLs found on this chromosome using several rat models (Joe and Garrett 2005). Multiple-strain comparisons have identified QTLs on this chromosome (Clemitson et al. 2002, 2007; Cui et al. 2003, 2004; Frantz et al. 1998, 2001; Hubner et al. 1999; Iwai et al. 1998; Joe et al. 2003; Kato et al. 2003; Kloting et al. 1998; Monti et al. 2003; Saad et al. 1999, 2001; St Lezin et al. 1999; Yagil et al. 2003; Yao et al. 2007). Of the three RNO1 BP QTLs reported from our laboratory (Saad et al. 2001), the present study focused on QTL1b. A new iteration of congenic substrains containing shorter introgressed segments spanning QTL1b were constructed and studied for BP effect. Data from two congenic substrains with nonoverlapping congenic regions indicated that the original 20,899,822-bp QTL-containing region contained at least two closely linked but separate genetic determinants. The combined effect of the QTL1b1 region and the QTL1b2 region was nonadditive, that is, the statistical significance of the sum of the effects of the two QTLs is smaller than what would have been expected if the BP effect of the two QTLs were additive. From the point of view of conferring resistance to the development of high $\mathrm{BP}$, such an interaction is unfavorable. This type of interaction is different from two other genetic interactions reported from our laboratory wherein the interaction results in an amplified BP effect: (1) Epistatic effect of BP QTLs on RNO10 and $\mathrm{RNO} 2$, wherein the sum effect of protective alleles (against high BP) at the two loci was greater than the effects of each 
Fig. 3 Phenotypic measurements by radiotelemetry. Rats were implanted with radiotelemetry probes as described in the Materials and methods section. Lines connecting the closed circles represent the BP of S rats. Lines connecting the open circles represent the $\mathrm{BP}$ of the congenic substrain

S.LEW(D1Mco36-D1Mco101). Data points represent the average values of 4 -h intervals. ${ }^{*} p<0.0001$ compared to $\mathrm{S}$. $\mathrm{SBP}=$ systolic blood pressure; $\mathrm{DBP}=$ diastolic blood pressure; $\mathrm{PP}=$ pulse pressure; $\mathrm{HR}=$ heart rate; $\mathrm{bpm}=$ beats $/$ $\min$
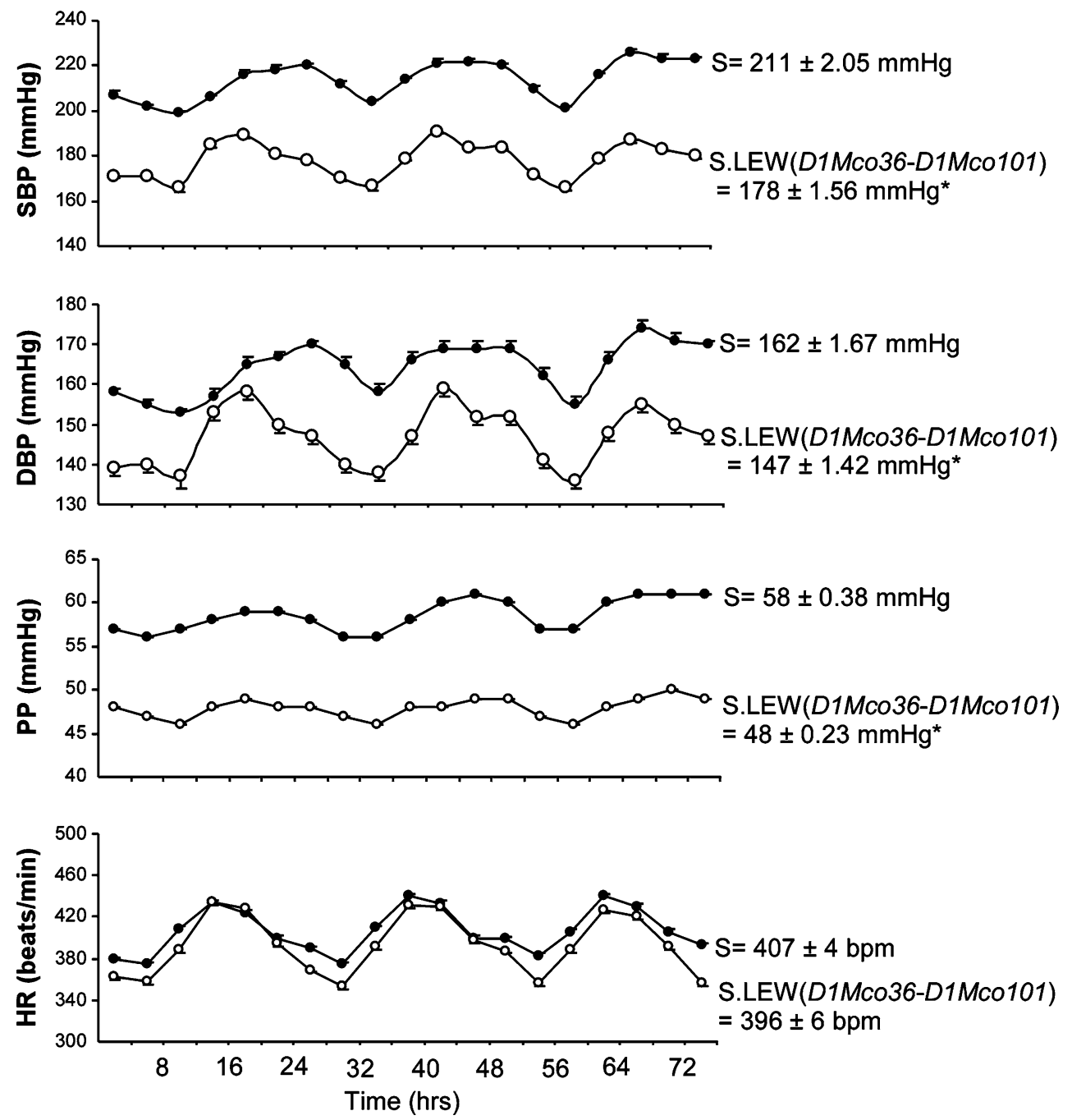

individual QTL (Rapp et al. 1998) and (2) interaction between the two QTLs on RNO5 wherein protective alleles (against high BP) at each locus was not sufficient by itself to demonstrate a BP-lowering effect but required the presence of protective alleles (against high $\mathrm{BP}$ ) from a closely linked interactive locus (Garrett and Rapp 2002). In the interest of mapping genetic elements that control BP, understanding that originally identified QTL regions are fragmented arrays of multiple genetic factors that may or may not interact with each other is important. For example, in another recent study from our laboratory, a region as small as $1.35 \mathrm{Mb}$ was found to harbor as many as at least three distinct but opposing genetic elements that control BP (Saad et al. 2007a, b).

Although it is helpful to know that multiple genetic determinants underlie a BP QTL, this evidence alone is not enough to prioritize candidate gene analysis. One of the common ways to prioritize candidate gene analysis is through the detection of single nucleotide polymorphisms (SNPs) as candidate quantitative trait nucleotides. Of the
19 genes contained within the QTL1b1 region, there is only one coding-region SNP between the SS/Jr and LEW/ $\mathrm{Crl}$ strains (http://www.ensembl.org). This variant is located within LOC680906, which is a gene with unknown function. Similarly, of the 81 genes within the QTL1b2 region, there are a total of 7 SNPs within the following genes: Rhesus blood group-associated C glycoprotein, alanyl (membrane) aminopeptidase, protein regulator of cytokinesis 1 (predicted), Unc-45 homolog A, adaptorrelated protein complex 3 , beta 2 subunit (predicted), BTB (POZ) domain containing 1, and ADAMTS-like 3 (predicted). None of these genes has been previously implicated in the control of blood pressure and therefore represent novel candidates for BP control.

A number of complementary techniques have been applied to expedite QTL gene identification (Arbilly et al. 2006; Cervino et al. 2007; Cowley 2006; Darvasi 2003; DiPetrillo et al. 2005; Glazier et al. 2002; Joe et al. 2005; Liang et al. 2003; Pravenec et al. 2001). Detection of cisacting expression QTLs (eQTLs) is one way of prioritizing 
Table 2 Real-time RT-PCR of genes within QTL1b1 and transcription factor genes outside of QTL1b1 and QTL1b2

\begin{tabular}{|c|c|c|c|}
\hline Gene name ${ }^{a}$ & GenBank Accession No. & S.LEW(D1Mco36-D1Mco101)-LS/S-LS ${ }^{c}$ & S.LEW(D1Mco36-D1Rat49)-LS/S-LS \\
\hline \multicolumn{4}{|c|}{ Genes within the QTL1b1 region: } \\
\hline Hypothetical protein & BE108294 & $0.76 \pm 0.03^{*}$ & $2.19 \pm 0.19^{*}$ \\
\hline Unknown & X62953 & $1.42 \pm 0.02^{*}$ & $1.99 \pm 0.20^{*}$ \\
\hline Chd 2 & NM_001271/XM_218790 & $1.48 \pm 0.05^{*}$ & $1.51 \pm 0.09^{*}$ \\
\hline Hypothetical protein & XM_980342 & $0.66 \pm 0.03^{*}$ & $0.70 \pm 0.02^{*}$ \\
\hline$N r 2 f 2$ & NM_080778 & $0.74 \pm 0.02^{*}$ & $2.14 \pm 0.11^{*}$ \\
\hline \multicolumn{4}{|c|}{ Transcription factor genes outside the QTL1b1 and QTL1b2 regions: } \\
\hline Gli & XM_345832 & $1.15 \pm 0.09$ & $2.04 \pm 0.23^{*}$ \\
\hline$H n f 4 a$ & NM_022180 & $0.93 \pm 0.04$ & $0.54 \pm 0.07^{*}$ \\
\hline Jun & NM_021835 & $1.74 \pm 0.20^{*}$ & $0.61 \pm 0.06^{*}$ \\
\hline$N r 4 a 1$ & NM_024388 & $1.67 \pm 0.15^{*}$ & $0.58 \pm 0.07^{*}$ \\
\hline p53 & NM_030989 & $0.77 \pm 0.02^{*}$ & $0.64 \pm 0.09^{*}$ \\
\hline Pcbd & M83740 & $0.53 \pm 0.04^{*}$ & $0.42 \pm 0.07^{*}$ \\
\hline Pparg & NM_013124 & $1.13 \pm 0.07$ & $0.42 \pm 0.07^{*}$ \\
\hline Rara & NM_031528 & $0.75 \pm 0.03^{*}$ & $0.55 \pm 0.06^{*}$ \\
\hline Rxra & NM_012805 & $0.61 \pm 0.03^{*}$ & $0.46 \pm 0.05^{*}$ \\
\hline$T c f 1$ & NM_012669 & $0.79 \pm 0.01^{*}$ & $0.59 \pm 0.14^{*}$ \\
\hline$T c f 2$ & NM_013103 & $0.72 \pm 0.03^{*}$ & $0.58 \pm 0.05^{*}$ \\
\hline Zfpm2 & XM_235253 & $0.74 \pm 0.01^{*}$ & $1.73 \pm 0.15^{*}$ \\
\hline
\end{tabular}

$\mathrm{S}=$ Dahl salt-sensitive; $\mathrm{LS}=$ low salt

* Significant differential expression $(p<0.05)$

a Gene name abbreviations: $N r 2 f 2$, nuclear receptor subfamily 2, group F, member2; $N r 4 a 1$, nuclear receptor subfamily 4 group A member 1 ; $P c b d$, 6-pyruvoyl-tetrahydropterin synthase/dimerization cofactor of $T c f 1$; Rara, retinoic acid receptor alpha; Rxra, retinoic X receptor alpha; $T c f 1$, transcription factor 1 (hepatic nuclear factor 1); $T c f 2$, transcription factor 2 (hepatic nuclear factor 2); Zfpm2, zinc finger protein multitype 2.

b Accession number correspond to mouse sequence. All other accessions correspond to rat sequences

${ }^{c}$ Numbers in column 3 are the mean ratio values \pm SE of 8 independent experiments performed in duplicate

candidate gene analysis (Hubner et al. 2005; Petretto et al. 2006a, b). This approach has been applied extensively to study the inheritance of gene expression differences in inbred, recombinant-inbred, consomic, and congenic rat strains (Aitman et al. 1999, Cicila and Lee 1998; Hubner et al. 2005; Lee and Cicila 2002; Lee et al. 2003, 2007; Liang et al. 2002; Malek et al. 2006; Petretto et al. 2006a, b). Expression QTLs are proposed to represent prototypical targets for a new class of transcription-modulating drugs aimed at treating patients with hypertension and metabolic syndrome (Pravenec and Kurtz 2007). However, further proof for eQTLs as positional candidates is obtained by retaining such cis-acting eQTLs within progressively shorter introgressed segments of congenic substrains that demonstrate a phenotypic effect, in our case, on BP. The differential expression of candidate genes such as $\mathrm{Nr} 2 \mathrm{f} 2$ or BE108294 being in one direction in a congenic strain with a longer introgressed segment and in the opposite direction in a congenic strain with a shorter introgressed segment implies that the expression of these genes is potentially influenced by unknown genetic factors that are closely linked. If the differential expression, regardless of the direction of change, was abolished in the congenic strain with the shorter introgressed segment, then one could conclude that gene expression of Nr2f2 or BE108294 is not the causal element for the observed BP effect elicited by QTL1b1. However, this is not the case. The differential expression of these positional candidate genes remains as a possible contributor to the observed QTL effect of QTL1b1. Conclusive evidence is thus warranted, which can be achieved only by further genetic dissection of this region. Thus, newer iterations of congenic substrains harboring shorter segments flanking $N r 2 f 2$ are required to further localize the causative gene(s) and to validate $N r 2 f 2$ and/or other differentially expressed positional candidate genes as BP QTLs.

Mapping quantitative trait loci using congenic strains is a time-consuming but time-tested approach to attach physiology to the genome (Jacob and Kwitek 2002; Joe and Garrett 2005, 2006; Joe et al. 2003). The current genetic dissection is certainly an intermediary but obligatory step toward achieving the ultimate goal of identifying the underlying genetic elements that are responsible for the observed alterations in BP. In addition, this information 
may be beneficial to further dissect the human BP QTLs described on the homologous region of QTL1b (Krushkal et al. 1999; Weder et al. 2003; Xu et al. 1999a, b), i.e., human chromosome 15 , which encompass larger genomic segments than the rat BP QTL1b and require further genetic dissection to delineate the number and magnitude of change imparted by the underlying genetic determinants of BP.

Acknowledgments Funding from NHLBI/NIH (RO1HL075414) to B. Joe and N. H. Lee is gratefully acknowledged. The authors thank John P. Rapp and George T. Cicila for critical comments on the manuscript.

\section{References}

Aitman TJ, Glazier AM, Wallace CA, Cooper LD, Norsworthy PJ et al (1999) Identification of $C d 36$ (Fat) as an insulin-resistance gene causing defective fatty acid and glucose metabolism in hypertensive rats. Nat Genet 21:76-83

Arbilly M, Pisante A, Devor M, Darvasi A (2006) An integrative approach for the identification of quantitative trait loci. Anim Genet 37(Suppl 1):7-9

Cervino AC, Darvasi A, Fallahi M, Mader CC, Tsinoremas NF (2007) An integrated in silico gene mapping strategy in inbred mice. Genetics 175:321-333

Cicila GT, Lee SJ (1998) Identifying candidate genes for blood pressure quantitative trait loci using differential gene expression and a panel of congenic strains. Hypertens Res 21:289-296

Clemitson JR, Pratt JR, Frantz S, Sacks S, Samani NJ (2002) Kidney specificity of rat chromosome 1 blood pressure quantitative trait locus region. Hypertension 40:292-297

Clemitson JR, Dixon RJ, Haines S, Bingham AJ, Patel BR et al (2007) Genetic dissection of a blood pressure quantitative trait locus on rat chromosome 1 and gene expression analysis identifies SPON1 as a novel candidate hypertension gene. Circ Res 100:992-999

Cowley AW Jr (2006) The genetic dissection of essential hypertension. Nat Rev Genet 7:829-840

Cui ZH, Ikeda K, Kawakami K, Gonda T, Nabika T et al (2003) Exaggerated response to restraint stress in rats congenic for the chromosome 1 blood pressure quantitative trait locus. Clin Exp Pharmacol Physiol 30:464-469

Cui ZH, Ikeda K, Kawakami K, Gonda T, Masuda J et al (2004) Exaggerated response to cold stress in a congenic strain for the quantitative trait locus for blood pressure. J Hypertens 22:21032109

Darvasi A (2003) Genomics: Gene expression meets genetics. Nature 422:269-270

Deng AY (2007) Genetic basis of polygenic hypertension. Hum Mol Genet 16:R195-202

DiPetrillo K, Wang X, Stylianou IM, Paigen B (2005) Bioinformatics toolbox for narrowing rodent quantitative trait loci. Trends Genet 21:683-692

Flint J, Mott R (2001) Finding the molecular basis of quantitative traits: successes and pitfalls. Nat Rev Genet 2:437-445

Frantz SA, Kaiser M, Gardiner SM, Gauguier D, Vincent $M$ et al (1998) Successful isolation of a rat chromosome 1 blood pressure quantitative trait locus in reciprocal congenic strains. Hypertension 32:639-646

Frantz S, Clemitson JR, Bihoreau MT, Gauguier D, Samani NJ (2001) Genetic dissection of region around the $\mathrm{Sa}$ gene on rat chromosome 1: evidence for multiple loci affecting blood pressure. Hypertension 38:216-221

Garrett MR, Rapp JP (2002) Two closely linked interactive blood pressure QTL on rat chromosome 5 defined using congenic Dahl rats. Physiol Genomics 8:81-86

Garrett MR, Meng H, Rapp JP, Joe B (2005) Locating a blood pressure quantitative trait locus within $117 \mathrm{~kb}$ on the rat genome: substitution mapping and renal expression analysis. Hypertension 45:451-459

Glazier AM, Nadeau JH, Aitman TJ (2002) Finding genes that underlie complex traits. Science 298:2345-2349

Gu L, Dene H, Deng AY, Hoebee B, Bihoreau M-T et al (1996) Genetic mapping of two blood pressure quantitative trait loci on rat chromosome 1. J Clin Invest 97:777-778

Hubner N, Lee YA, Lindpaintner K, Ganten D, Kreutz R (1999) Congenic substitution mapping excludes $\mathrm{Sa}$ as a candidate gene locus for a blood pressure quantitative trait locus on rat chromosome 1. Hypertension 34:643-648

Hubner N, Wallace CA, Zimdahl H, Petretto E, Schulz H et al (2005) Integrated transcriptional profiling and linkage analysis for identification of genes underlying disease. Nat Genet 37:243-253

Iwai N, Tsujita Y, Kinoshita M (1998) Isolation of a chromosome 1 region that contributes to high blood pressure and salt sensitivity. Hypertension 32:636-638

Jacob HJ, Kwitek AE (2002) Rat genetics: attaching physiology and pharmacology to the genome. Nat Rev Genet 3:33-42

Joe B, Garrett MR (2005) Substitution mapping: Using congenic strains to detect genes controlling blood pressure. In: Raizada MK, Kasparov S, Katovich MJ (eds) Cardiovascular Genomics. Totowa, NJ, Humana Press pp 41-58

Joe B, Garrett MR (2006) Genetic analysis of inherited hypertension in the rat. In: Dominiczak A, Connell J (eds) Genetics of Hypertension. Amrsteram, The Netherlands, Elsevier Science pp 177-200

Joe B, Garrett MR, Dene H, Rapp JP (2003) Substitution mapping of a blood pressure quantitative trait locus to a $2.73 \mathrm{Mb}$ region on rat chromosome 1. J Hypertens 21:2077-2084

Joe B, Letwin NE, Garrett MR, Dhindaw S, Frank B et al (2005) Transcriptional profiling with a blood pressure QTL intervalspecific oligonucleotide array. Physiol Genomics 23:318-326

Kato N, Nabika T, Liang Y-Q, Mashimo T, Inomata H et al (2003) Isolation of a chromosome region affecting blood pressure and vascular disease traits in the stroke-prone rat model. Hypertension 42:1191-1197

Kloting I, Voigt B, Kovacs P (1998) Metabolic features of newly established congenic diabetes-prone BB.SHR rat strains. Life Sci 62:973-979

Krushkal J, Ferrell R, Mockrin SC, Turner ST, Sing CF et al (1999) Genome-wide linkage analyses of systolic blood pressure using highly discordant siblings. Circulation 99:1407-1410

Lee SJ, Cicila GT (2002) Functional genomics in rat models of hypertension: using differential expression and congenic strains to identify and evaluate candidate genes. Crit Rev Eukaryot Gene Expr 12:297-316

Lee SJ, Liu J, Qi N, Guarnera RA, Lee SY et al (2003) Use of a panel of congenic strains to evaluate differentially expressed genes as candidate genes for blood pressure quantitative trait loci. Hypertens Res 26:75-87

Lee SJ, Liu J, Westcott AM, Vieth JA, DeRaedt SJ et al (2006) Substitution mapping in Dahl rats identifies two distinct blood pressure quantitative trait loci within $1.12-$ and $1.25-\mathrm{Mb}$ intervals on chromosome 3. Genetics 174:2203-2213

Lee NH, Haas BJ, Letwin NE, Frank BC, Luu TV et al (2007) Crosstalk of expression quantitative trait loci within two interacting blood pressure quantitative trait loci. Hypertension 50:11261133 
Liang M, Yuan B, Rute E, Greene AS, Zou AP et al (2002) Renal medullary genes in salt-sensitive hypertension: a chromosomal substitution and cDNA microarray study. Physiol Genomics 8:139-149

Liang M, Yuan B, Rute E, Greene AS, Zou AP et al (2003) Insights into Dahl salt-sensitive hypertension revealed by temporal patterns of renal medullary gene expression. Physiol Genomics 12:229-237

Malek RL, Wang HY, Kwitek AE, Greene AS, Bhagabati N et al (2006) Physiogenomic resources for rat models of heart, lung and blood disorders. Nat Genet 38:234-239

Meng H, Garrett MR, Dene H, Rapp JP (2003) Localization of a blood pressure QTL to a 2.4-cM interval on rat chromosome 9 using congenic strains. Genomics 81:210-220

Monti J, Plehm R, Schulz H, Ganten D, Kreutz R et al (2003) Interaction between blood pressure quantitative trait loci in rats in which trait variation at chromosome 1 is conditional upon a specific allele at chromosome 10. Hum Mol Genet 12:435-439

Petretto E, Mangion J, Dickens NJ, Cook SA, Kumaran MK et al (2006a) Heritability and tissue specificity of expression quantitative trait loci. PLoS Genet 2:e172

Petretto E, Mangion J, Pravanec M, Hubner N, Aitman TJ (2006b) Integrated gene expression profiling and linkage analysis in the rat. Mamm Genome 17:480-489

Pravenec M, Kurtz TW (2007) Molecular genetics of experimental hypertension and the metabolic syndrome. From gene pathways to new therapies. Hypertension 49:941-952

Pravenec M, Landa V, Zidek V, Musilova A, Kren V et al (2001) Transgenic rescue of defective CD36 ameliorates insulin resistance in spontaneously hypertensive rats. Nat Genet 27:156-158

Rapp JP, Garrett MR, Deng AY (1998) Construction of a double congenic strain to prove an epistatic interaction on blood pressure between rat chromosomes 2 and 10. J Clin Invest 101:1591-1595

Saad Y, Garrett MR, Lee SJ, Dene H, Rapp JP (1999) Localization of a blood pressure QTL on rat chromosome 1 using Dahl rat congenic strains. Physiol Genomics 1:119-125
Saad Y, Garrett MR, Rapp JP (2001) Multiple blood pressure QTL on rat chromosome 1 defined by Dahl rat congenic strains. Physiol Genomics 4:201-214

Saad Y, Garrett MR, Manickavasagam E, Yerga-Woolwine S, Farms $\mathrm{P}$ et al (2007a) Fine-mapping and comprehensive transcript analysis reveals nonsynonymous variants within a novel $1.17 \mathrm{Mb}$ blood pressure QTL region on rat chromosome 10. Genomics 89:343-353

Saad Y, Yerga-Woolwine S, Saikumar J, Farms P, Manickavasagam E et al (2007b) Congenic interval mapping of RNO10 reveals a complex cluster of closely-linked genetic determinants of blood pressure. Hypertension 50:891-898

St Lezin E, Griffin KA, Picken M, Churchill MC, Churchill PC et al (1999) Genetic isolation of a chromosome 1 region affecting susceptibility to hypertension-induced renal damage in the spontaneously hypertensive rat. Hypertension 34:187-191

Stoll M, Kwitek-Black AE, Cowley AW Jr, Harris EL, Harrap SB et al (2000) New target regions for human hypertension via comparative genomics. Genome Res 10:473-482

Weder AB, Delgado MC, Zhu X, Gleiberman L, Kan D et al (2003) Erythrocyte sodium-lithium countertransport and blood pressure: a genome-wide linkage study. Hypertension 41:842-846

Xu X, Rogus JJ, Terwedow HA, Yang J, Wang Z et al (1999a) An extreme-sib-pair genome scan for genes regulating blood pressure. Am J Hum Genet 64:1694-1701

Xu X, Yang J, Rogus J, Chen C, Schork N (1999b) Mapping of a blood pressure quantitative trait locus to chromosome $15 \mathrm{q}$ in a Chinese population. Hum Mol Genet 8:2551-2555

Yagil C, Hubner N, Kreutz R, Ganten D, Yagil Y (2003) Congenic strains confirm the presence of salt-sensitivity QTLs on chromosome 1 in the Sabra rat model of hypertension. Physiol Genomics 12:85-95

Yao H, Cui ZH, Masuda J, Nabika T (2007) Congenic removal of a QTL for blood pressure attenuates infarct size produced by middle cerebral artery occlusion in hypertensive rats. Physiol Genomics 30:69-73 